\title{
Phosphatidylthreonine: An exclusive phospholipid regulating calcium homeostasis and virulence in a parasitic protist
}

\author{
Ruben D. Arroyo-Olarte and Nishith Gupta* \\ Department of Molecular Parasitology, Humboldt University, Berlin, Germany. \\ * Corresponding Author: \\ Nishith Gupta, PhD, Department of Molecular Parasitology, Humboldt University, Philippstrasse 13, House 14, Berlin, Germany; Tele- \\ phone: +49-30-20936404; Fax: +49-30-20936051; E-mail: Gupta.Nishith@staff.hu-berlin.de
}

\begin{abstract}
Lipids are often overlooked for their functional relevance in the biological membranes, while membrane-bound proteins with their great diversity of pumps, channels and enzymes are seen as the major driving force for most dynamic processes occurring in biomembranes. Lipids are in fact much more versatile than a mere physical support for biomembranes or a rich source of energy for cells. From cholesterol, determining the assembly of integral membrane proteins in the lipid rafts, to diacylglycerol and inositides as the key secondary messengers, the diversity of lipid functions is constantly being discovered. Even more unknown is the role of those non-customary, minor lipids. One such bizarre lipid is phosphatidylthreonine (PtdThr), originally described as a byproduct of mammalian phosphatidylserine (PtdSer) synthase, when threonine was used as an alternative substrate (instead of serine) for the baseexchange reaction with phosphatidylcholine (PtdCho) or phosphatidylethanolamine (PtdEtn). In our lately published work [1], we surprisingly identified PtdThr as the major and natural phospholipid in a prevalent protozoan parasite Toxoplasma gondii, where it was even three to four times more abundant than the prototypical PtdSer.
\end{abstract}

To reveal the origin of this unusual phospholipid we examined the two putative base-exchange-type lipid synthases encoded by the parasite genome. When expressed in $E$. coli, both enzymes used serine as a substrate to produce PtdSer, but only one of them, henceforth called PtdThr synthase (TgPTS), was able to readily utilize threonine into PtdThr. When the TgPTS gene was disrupted, not only the de novo synthesis of PtdThr was abolished, but also the amount of PtdSer was proportionally increased in the mutant parasites. Moreover, complementation of the mutant with an exogenous gene copy of TgPTS reinstated PtdThr and reversed PtdSer to a normal level. Physiological relevance of the catalytic activity of TgPTS was confirmed by expressing a TgPTS isoform containing a deletion in the predicted base-exchange domain. The results also indicated that TgPTS could generate PtdThr by a baseexchange mechanism similar to the one used by the mammalian PtdSer synthases to make PtdSer. TgPTS and its orthologs in selected Chromalveolates (Neospora, Eimeria and Phytophthora) contain a series of mutations in the vicinity of the conserved substrate binding pocket when compared to the mainstream PtdSer synthases including TgPSS. These residues may be critical to a divergent evolution of these two enzymes.

But, what is the importance of PtdThr and can PtdSer replace its physiological function in $T$. gondii? To address these questions we resorted to a comprehensive analysis of the parasite's lytic cycle, i.e. the successive steps of intracellular replication, egress and ensuing invasion of fresh host cells. Surprisingly, the loss of PtdThr in the $\Delta$ tgpts mutant did not affect the parasite replication, suggesting that PtdThr is dispensable for membrane biogenesis and that it can be readily replaced by its near-universal analog PtdSer in this regard. But then, why did $T$. gondii invent an enzymatic pathway to produce such a peculiar lipid? The answer lies in the other two steps of the lytic cycle, egress and invasion, both showing a noteworthy impairment in the $\Delta$ tgpts mutant. Egress and invasion are active processes requiring motile parasites. Therefore, parasite motility was evaluated and, as expected, it was also blunted by the lack of PtdThr and recovered upon genetic complementation with a functional enzyme. Next, we investigated whether the observed in vitro defects in egress, invasion and motility impacted the parasite virulence in a murine model. Animals survived the infection with the $\Delta$ tgpts mutant, even at 100 -fold higher doses compared to control mice infected with the parental strain, which was explicitly 
A

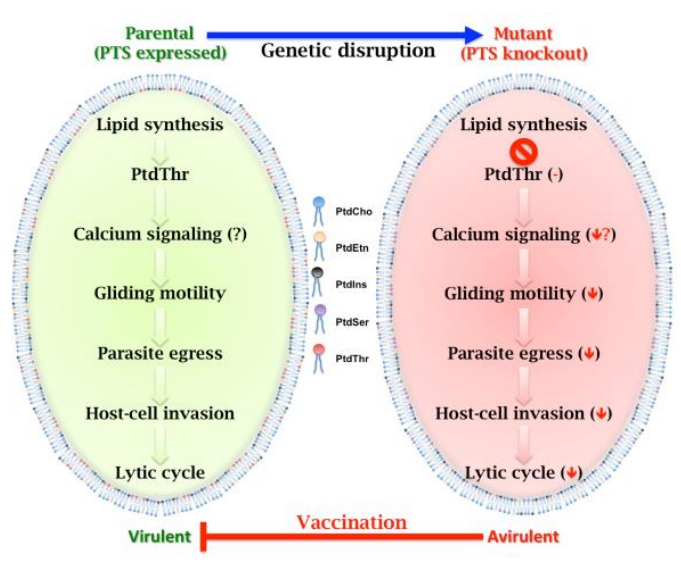

B

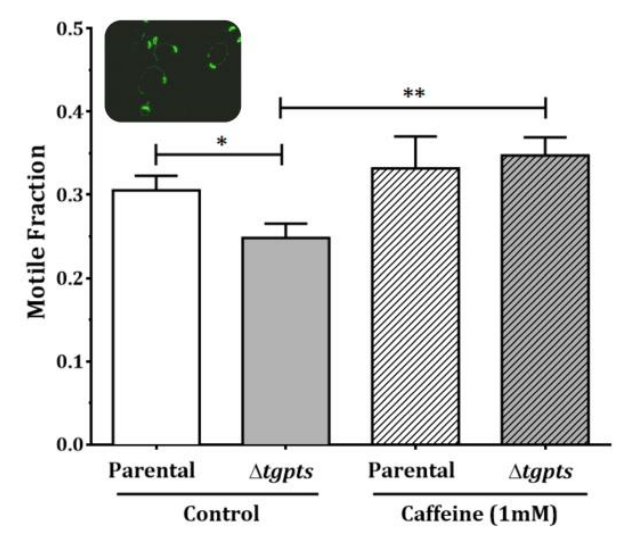

FIGURE 1: Phosphatidylthreonine-mediated regulation of calcium signaling and virulence in $T$. gondii. (A) Scheme illustrating the effect of PTS gene knockout on the parasite. Image is adapted from reference 2. (B) Motile fraction of the indicated parasite strains treated with or without caffeine. Numbers of protein trails (immunostained with anti-Sag1 antibody) formed by extracellular parasites were counted to estimate the motile fraction.

lethal. More importantly, a single-dose infection of mice with the PTS mutant made them fully resistant to an otherwise lethal challenge with a hypervirulent strain of $T$. gondii. Moreover, the mutant-immunized mice avoided the formation of brain cysts by a cyst-forming strain and were protected against the yet-incurable chronic infection. So it

\section{REFERENCES}

1. Arroyo-Olarte RD, Brouwers JF, Kuchipudi A, Helms JB, Biswas A, Dunay IR, Lucius R, Gupta N (2015). Phosphatidylthreonine and lipidmediated control of parasite virulence. PLoS Biol 13(11):e1002288.

2. Roberts RG (2015). Toxoplasma's taste for exotic fat. PLoS Biol 13(11):e1002289. appears as though $T$. gondii has evolved PtdThr during the course of evolution to optimize its parasitic lifestyle. Notably, as illustrated (Fig. 1), the pathway can be exploited to develop a metabolically attenuated vaccine.

Our study also elicited many questions, mainly regarding the lipid-mediated mechanisms regulating the lytic cycle of $T$. gondii. It has long been established that gliding motility, which underlies the observed defects in the mutant, is governed by calcium signaling. Therefore, it is likely that PtdThr influences calcium flux across the plasma membrane and/or calcium storage organelles, such as acidocalcisomes and endoplasmic reticulum. Indeed, incubation of PtdThr-deficient parasites with caffeine, a wellknown agonist of ryanodine receptors $/ \mathrm{Ca}^{2+}$ channels, restored the motile fraction to the parental level (Fig. 1B). Additional studies measuring calcium in intracellular parasites using an optogenetic sensor also suggest a role of PtdThr in calcium homeostasis [3]. Prospective work on the biophysical and biochemical features of PtdThr shall reveal further mechanistic insights into the functioning of this exclusive lipid in $T$. gondii.

\section{ACKNOWLEDGMENTS}

NG is grateful to German Research Foundation (DFG) for grants SFB618/C7 and GU1100/4-1 supporting this work.

\section{CONFLICT OF INTEREST}

The authors declare that there are no conflicts of interest pertaining to this work.

\section{COPYRIGHT}

(C) 2016 Arroyo-Olarte and Gupta. This is an open-access article released under the terms of the Creative Commons Attribution (CC BY) license, which allows the unrestricted use, distribution, and reproduction in any medium, provided the original author and source are acknowledged.

Please cite this article as: Ruben D. Arroyo-Olarte and Nishith Gupta (2016). Phosphatidylthreonine: An exclusive phospholipid regulating calcium homeostasis and virulence in a parasitic protist. Microbial Cell 3(5): 189-190.

3. Kuchipudi A, Arroyo-Olarte RD, Hoffmann F, Brinkmann V, Gupta N (2016). Optogenetic monitoring of the parasite calcium identifies a phosphatidylthreonine-regulated ion homeostasis in Toxoplasma gondii. Microbial Cell 3(5): 215-223. 